\title{
Primary mucinous adenocarcinoma of the eyelid: the first reported case in the Middle East
}

\begin{abstract}
Primary mucinous adenocarcinoma (PMA) is a rare malignant subtype of the sweat gland tumors that most often involves the periorbital region. Differentiation between PMA and metastatic mucinous adenocarcinoma based on histologic features alone is difficult. Therefore, immunohistochemical staining and cytokeratin profiles are essential. Isolated case reports or small series of periocular/eyelid PMA have been published, none of which is being reported in our geographical area. We are describing a 57-year-old patient with a confirmed diagnosis of lower eyelid PMA as the first reported case in the Middle East.
\end{abstract}

\author{
Volume 7 Issue 7 - 2017 \\ Hind Manaa Alkatan, ${ }^{1,2}$ Abdullah Ibrahim \\ Almater,' Hibah Abdulaziz Alowais, ${ }^{3}$ Osama \\ Al Sheikh, ${ }^{4}$ Azza MY Maktabi ${ }^{5}$ \\ 'College of Medicine, King Saud University, Saudi Arabia \\ ${ }^{2}$ Departments of Ophthalmology \& Pathology, King Saud \\ University-Medical City, Saudi Arabia \\ ${ }^{3}$ College of Medicine, Almaarefa Colleges, Saudi Arabia \\ ${ }^{4}$ Oculoplastics and Orbit Division, King Khaled Eye Specialist \\ Hospital, Saudi Arabia \\ ${ }^{5}$ Pathology \& Laboratory Medicine Department, King Khaled \\ Eye Specialist Hospital, Saudi Arabia
}

Correspondence: Hind Manaa Alkatan, Assistant Professor, College of Medicine, King Saud University, Chief of Ophthalmic Pathology Division, Consultant: Departments of Ophthalmology and Pathology, King Abdulaziz University Hospital, Riyadh, Saudi Arabia, Director of KSU Residency, Training Program in Ophthalmology, College of Medicine, King Saud University, Riyadh, Saudi Arabia, PO Box 18097, Riyadh, postal code II4I5, Saudi Arabia, Tel +966504492399,

Email hindmkatan@gmail.com, hindkatan@yahoo.com

Received: November 28, 2017| Published: December 08, 2017

Abbreviations: PMA, primary mucinous adenocarcinoma; IHC, Immunohistochemical; CK7, Cytokeratin 7; EMA, epithelial membrane antigen; ER, estrogen receptor; $\mathrm{PR}$, progesterone receptor; CEA, carcinoembryonic antigen; CK20, cytokeratin 20; PAS, periodic acid schiff

\section{Introduction}

Primary mucinous adenocarcinoma (PMA) is a rare malignant subtype of the sweat gland tumors that most often involves the periorbital region. This tumor often presents as an asymptomatic, slow-growing mass that is usually mistaken for a benign lesion. ${ }^{1-3}$ It is difficult to differentiate PMA from metastatic mucinous adenocarcinoma based on histologic features alone. For that reason, immunohistochemical (IHC) staining and cytokeratin profiles have been studied to aid in the differentiation between the two. We are presenting a case of a 57-year-old male with a slowly growing eyelid mass that clinically resembled an intradermal nevus. In addition, we will highlight the important clinical, histological and immunohistochemical diagnostic features of this tumor.

\section{Case report}

A 57-year-old male, presented to the oculoplastic clinic with a 2-years history of progressive slowly growing slightly pigmented left lower eyelid mass that was associated with occasional discharge and has been clinically thought to be a benign nevus. The patient denied any history of surgery or trauma related to the site of the lesion. There was no history of pain or visual disturbance. The patient had systemic diabetes mellitus, hyperlipidemia and bronchial asthma.

External examination demonstrated a soft pedunculated mass measuring $13 \times 11 \mathrm{~mm}$ involving the outer $1 / 3$ of the left lower eyelid margin and extending to the lateral canthus (Figure 1A). Feeder vessels and focal pigmentation was observed. There was no ulceration, induration, nor signs of infection. No palpable regional lymph nodes were found. The rest of his ocular examination including the visual acuity was unremarkable in both eyes. The patient underwent excisional biopsy of the lesion for tissue diagnosis. The excised tumor grossly was rounding, tan-colored and firm in consistency. It measured 13 X 11 X 8 mm. Histopathologically; the mass was lined externally by unremarkable epidermis. The dermis disclosed a nodular mass consisting of tumor cells proliferating with tubular and cribriform patterns in a mucinous background (Figure 1B\&C). Some of the cells were bland in appearance, however islands of malignant dysplastic cells with prominent nucleoli and few mitoses were observed floating in mucinous lakes. The tumor cells were observed at the level of deep surgical margin. Extensive IHC stains were done. The tumor cells expressed Cytokeratin 7 (CK7) (Figure 1D), Epithelial membrane antigen (EMA), Estrogen receptor (ER), Progesterone receptor (PR), and Gross Cystic Disease Fluid Protein -15 (GCDFP-15) (Figure 2A-C). The tumor cells focally expressed Carcinoembryonic antigen (CEA), and P63 (Figure 2D), while the IHC staining was negative to Cytokeratin 20 (CK20). 


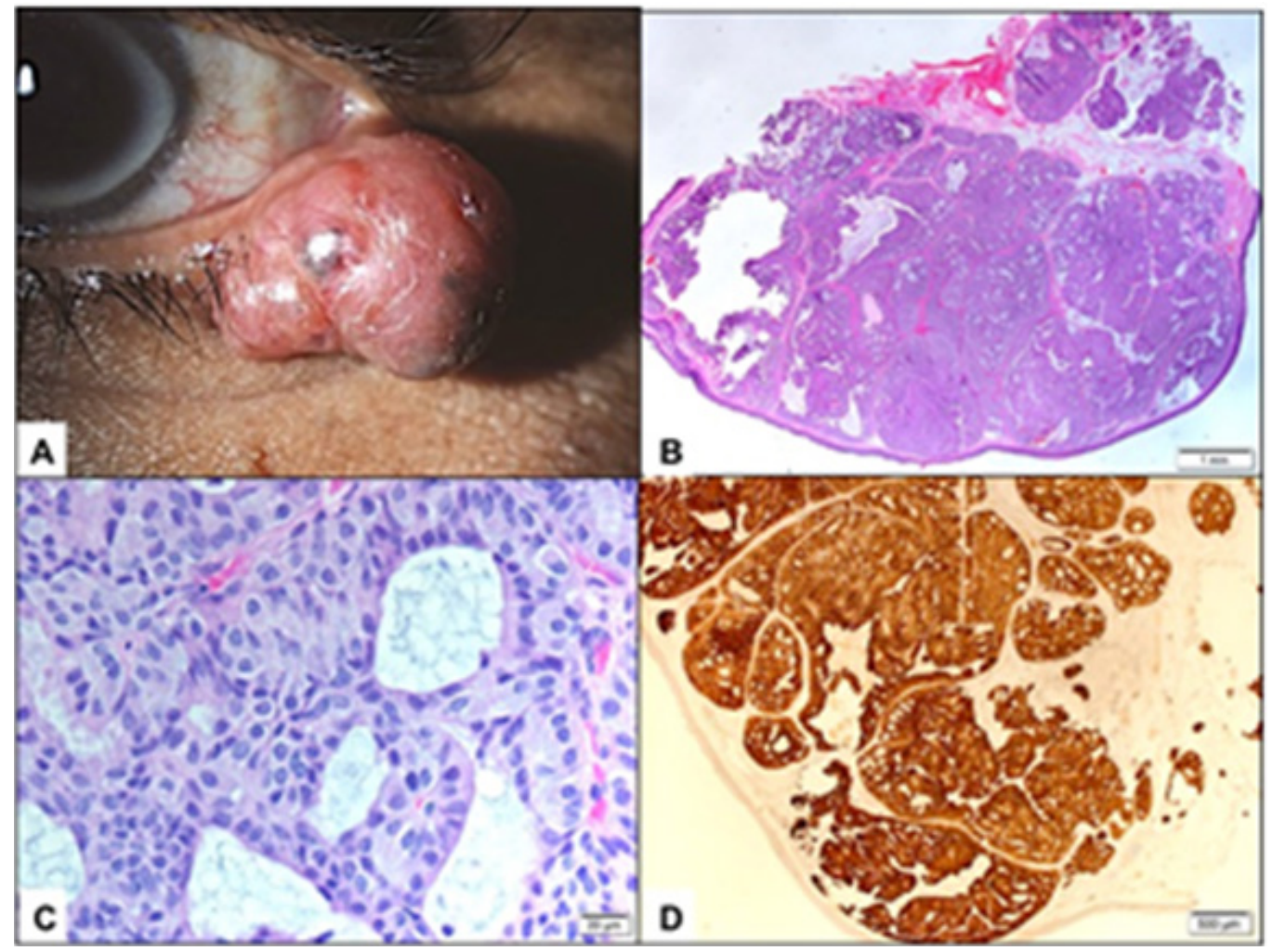

Figure I A The clinical appearance of the left lower eyelid lesion. B Lower power histopathological appearance of the lesion within the dermis (Original magnification X50 Hematoxylin \& Eosin). C The higher power appearance of theproliferatingtumor cells with cribriform pattern (Original magnification X400 Hematoxylin \& Eosin). D The tumor cells expressing Cytrokeratin-7 in all islands (Original magnification XI00 CK-7).

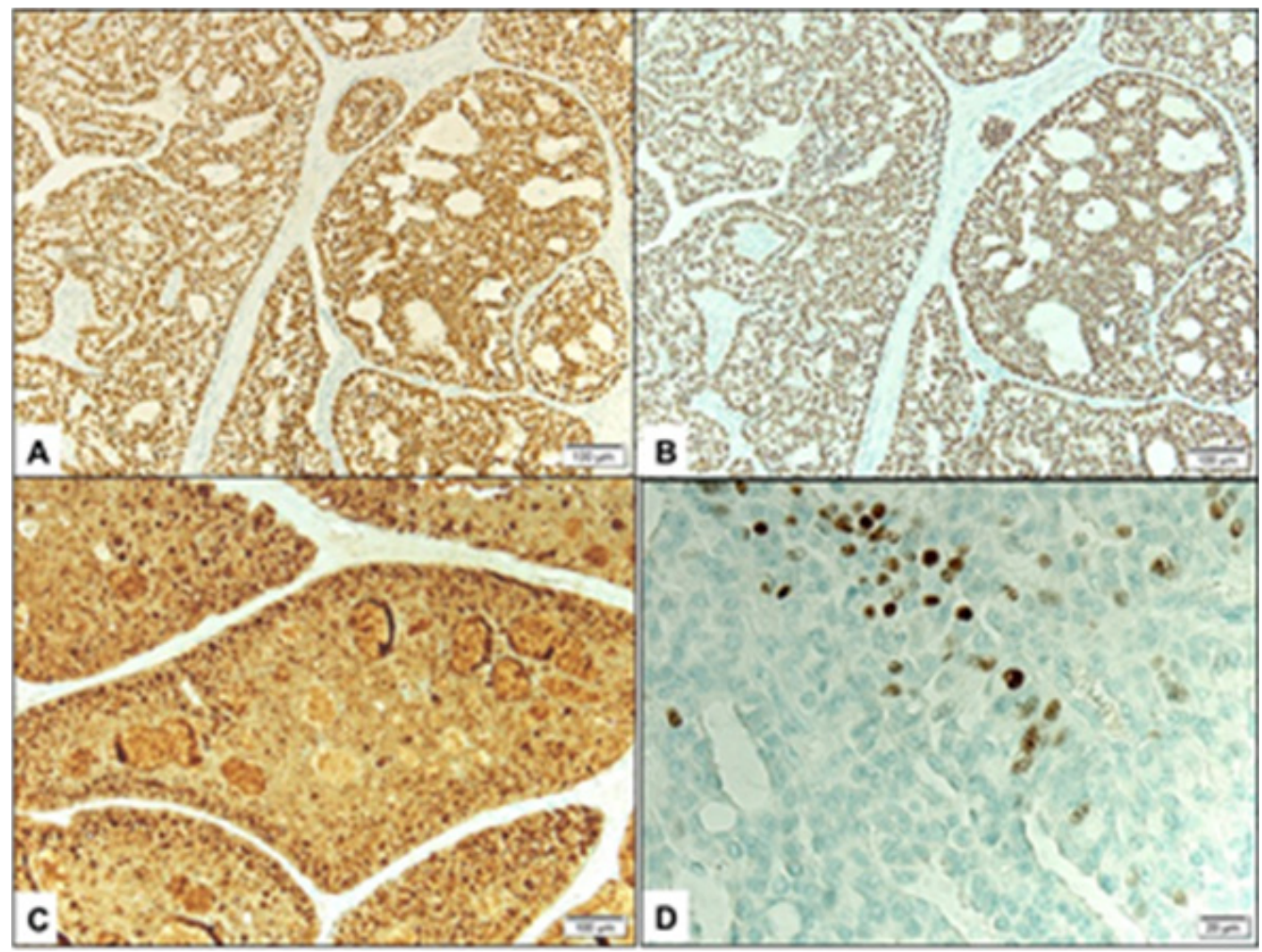

Figure 2A\&B Expression of Estrogen receptor (in A) and Progesterone receptor (in B) by the tumor cells (Original magnification XI00). C Expression of Gross Cystic Disease Fluid Protein by the tumor cells (Original magnification XI00 GCDFP-I5). D Nuclear expression of p63 by some tumor cells focally (Original magnification X400 p63). 
Complete systemic work-up excluded any primary source for metastasis. The overall histopathological and immunohistochemical findings were consistent with primary mucinous sweat gland adenocarcinoma with incomplete surgical margins of excision. Three weeks later, a definitive surgical re-excision with frozen section margin control was performed. The patient has been followed-up for nine months with satisfactory cosmetic result and no evidence of recurrence.

\section{Discussion}

Primary mucinous adenocarcinoma (PMA) was first described by Lennox et al. ${ }^{4}$ in 1952 as a rare histopathologic subtype of sweat gland tumors. PMA commonly arises in the head or neck, with the eyelid being the most common site of presentation in 40 percent of reported cases. $^{5}$ In 1979, Wright and Font's reported the largest case series of 21 cases of eyelid PMA [1]. Men are more commonly affected than female with a $2: 1$ ratio. ${ }^{1,5,6}$ However, in a recent smaller case series of 6 lesions, the tumor was more common in women than men, which could be related to the smaller sample size. ${ }^{2}$ It tends generally to occur in elderly individuals, with an average age of onset of 61.3 years (range 30-87 years). ${ }^{6}$ But, it has been reported in a 14-years-old girl. ${ }^{7}$ Upper eyelid seems to be less affected in $38-50 \%$ of the cases. ${ }^{2,6}$ Clinically, PMA has an indolent course with no predisposing factors. The clinical presentation of PMA can vary from case to case, that is why the careful histopathologic examination is necessary in the diagnosis of this tumor. In many cases, the lesion is clinically diagnosed as an epidermoid cyst, papilloma, lipoma, neoplasm, chalazion, pyogenic granuloma, hemangioma, sebaceous cyst, carcinoma (squamous cell carcinoma, or basal cell carcinoma), malignant melanoma and cutaneous metastasis. ${ }^{1-3}$

Classically, the histopathological features include dermal infiltration with nests of tumor cells arranged in glandular, cribriform or micro-papillary patterns surrounded by lakes of mucin and separated by strands of fibrous septa. ${ }^{1,2,5,6,8}$ The neoplastic cells usually show mild nuclear pleomorphism with few mitotic figures, round to cuboidal nuclei, occasional prominent nucleoli, and scanty eosinophilic cytoplasm. ${ }^{1,5,8}$ The mucin produced by the tumor cells is Periodic acid Schiff (PAS) positive, and is highlighted using colloidal iron, mucicarmine and alcian blue (PH 2.4) stains, which is consistent with the presence of a non-sulfated sialomucin that is sialidase labile but hyaluronidase and diastase resistant. ${ }^{1,5}$ Theoretically, the slow growth and low metastatic rate of mucinous adenocarcinoma has been linked to this copious mucin secretion, which interferes with cellular nutrition, thereby hindering growth and differentiation of neoplastic cells. ${ }^{8}$ It can be very difficult to distinguish primary mucinous adenocarcinoma from metastatic tumor based on histopathologic appearance alone. However, metastatic tumors are expected to have more dysplastic features including: poorly organized epithelial cells, prominent hyperchromasia and more mitotic figures than what is observed in PMA. ${ }^{3}$

Immunohistochemical staining of primary mucinous adenocarcinoma has been studied in attempt to differentiate primary mucinous adenocarcinoma from metastatic mucinous adenocarcinoma. The tumor cells of primary mucinous adenocarcinoma are cytokeratin 7 (CK7) positive and cytokeratin 20 (CK20) negative, which is similar to breast adenocarcinoma. ${ }^{3,9,10}$ In contrast, gastrointestinal adenocarcinoma tumor cells are CK7 negative and CK20 positive.,
In addition, the histopathologic hint for tumor cells from an intestinal origin would be dirty necrosis (necrotic eosinophilic foci containing nuclear debris) and the presence of epithelial cells with goblet cell differentiation. ${ }^{9}$ Primary mucinous adenocarcinoma tumor cells also express epithelial membrane antigen (EMA), carcinoembryonic antigen (CEA), gross cystic disease fluid protein 15 (GCDFP $15)$, estrogen receptor (ER) and progesterone receptor (PR)..$^{2,3,10}$ Recently, several studies support the use of p63 as a marker for differentiating primary mucinous adenocarcinoma from metastatic adenocarcinoma. ${ }^{3,10,11}$ The expression of p63 confirms the presence of myoepithelial cells indicating a primary adnexal origin of mucinous adenocarcinoma. ${ }^{11}$ However, it has been as stated that full clinical investigation remains the gold-standard approach to establish the origin of primary mucinous adenocarcinoma. ${ }^{9}$ Our case demonstrated enough IHC staining properties to confirm the diagnosis of PMA. The patient also had a negative systemic work up for any primary malignancy elsewhere.

PMA has been identified as a locally aggressive tumor with a low metastatic rate. ${ }^{1}$ The treatment modality of choice for eyelid PMA is wide local excision. Recently, some studies have suggested Moh's micrographic surgery as an alternative method. ${ }^{2,5,12}$ The reported recurrence rate of PMA in previous studies is 26 to $40 \%{ }^{1,5,6,11,13}$ The high recurrence rate is usually related to incomplete resection and specific tumor locations that are challenging for excision such as lesions involving the inner canthus. ${ }^{14}$

\section{Conclusion}

We present a case of a 57-year-old male with a primary mucinous adenocarcinoma involving the lower eyelid. A high index of suspicion is required to diagnose this uncommon entity especially that it tends to mimic benign lesions clinically and can be confused with metastatic mucinous adenocarcinoma histopathologically. Immunohistochemical staining offers a promising tool for the differentiation between primary and metastatic lesions.

\section{Declaration statement}

The authors have no financial or conflict of interest related to this work. An informed General Consent has been taken, which includes using patient's anonymous information. This case report has been approved by the HEC and Research center at KKESH.

\section{Acknowledgments}

None.

\section{Conflicts of interest}

Author declares that there are no conflicts of interest.

\section{Funding}

None.

\section{References}

1. Wright JD, Font RL. Mucinous sweat gland adenocarcinoma of the eyelid. A clinicopathologic study of 21 cases with histochemical and electron microscopic observations. Cancer. 1979;44(5):1757-1768.

2. Zhang Q, Wojno TH, Fitch SD, et al. Mucinous eccrine adenocarcinoma of the eyelid: Report of 6 cases. Can J Ophthalmol. 2010;45(1):76-78. 
3. Sanft DM, Zoroquiain P, Arthurs B, et al. Primary Mucinous Adenocarcinoma of the eyelid: A Case-Series. Human Pathology: Case Reports. 2017;9:19-23.

4. Lennox B, Pearse AG, Symmers WS. The frequency and significance of mucin in sweat gland tumors. Br J Cancer. 1952;6(4):363-368.

5. Snow SN, Reizner GT. Mucinous eccrine carcinoma of the eyelid. Cancer. 1992;70:2099-2104.

6. Segal A, Segal N, Gal A, et al. Mucinous sweat gland adenocarcinoma of the eyelid - current knowledge of a rare tumor. Orbit. 2010;29(6):334340 .

7. Latorre A, Alghothani L, Lambert D, et al. Mucin-producing malignant tumor of lower eyelid presenting in a 14-year-old Patient. J Clin Aesthet Dermatol. 2012;5(4):44-47.

8. Hemalatha AL, Kausalya SK, Amita K, et al. Primary mucinous eccrine adenocarcinoma - a rare malignant cutaneous adnexal neoplasm at an unconventional site. J Clin Diagn Res. 2014;8(8):FD14-FDA15.

9. Kazakov DV, Suster S, LeBoit PE, et al. Mucinous carcinoma of the skin, primary and secondary: A clinicopathologic study of 63 cases with emphasis on the morphologic spectrum of primary cutaneous forms: homologies with mucinous lesions in the breast. Am J Surg Pathol. 2005;29(6):764-782.
10. Rollins-Raval M, Chivukula M, Tseng GC, et al. An immunohistochemical panel to differentiate metastatic breast carcinoma to skin from primary sweat gland carcinomas with a review of the literature. Arch Pathol Lab Med. 2011;135(8):975-983.

11. Papalas JA, Proia AD. Primary mucinous carcinoma of the eyelid: a clinicopathologic and immunohistochemical study of 4 cases and an update on recurrence rates. Arch Ophthalmol. 2010;128(9):1160-1165.

12. Bindra M, Keegan DJ, Guenther T, et al. Primary cutaneous mucinous carcinoma of the eyelid in a young male. Orbit. 2005;24(3):211-214.

13. Marra DE, Schanbacher CF, Torres A. Mohs micrographic surgery of primary cutaneous mucinous carcinoma using immunohistochemistry for margin control. Dermatol Surg. 2004;30(5):799-802.

14. Cohen KL, Peiffer RL, Lipper S. Mucinous sweat gland adenocarcinoma of the eyelid. Am J Ophthalmol. 1981;92(2):183-188. 